\title{
Expression of GLUT1 is associated with increasing grade of malignancy in non-invasive and invasive urothelial carcinomas of the bladder
}

\author{
HENNING REIS ${ }^{1}$, STEPHAN TSCHIRDEWAHN ${ }^{2}$, TIBOR SZARVAS ${ }^{2}$, HERBERT RÜBBEN ${ }^{2}$, \\ KURT WERNER SCHMID ${ }^{1}$ and FLORIAN GRABELLUS ${ }^{1}$ \\ ${ }^{1}$ Institute of Pathology and Neuropathology, and ${ }^{2}$ Clinic of Urology, \\ University Hospital of Essen, University of Duisburg-Essen, Essen, Germany
}

Received April 13, 2011; Accepted August 2, 2011

DOI: $10.3892 / \mathrm{ol} .2011 .394$

\begin{abstract}
Glucose Transporter 1 (GLUT1) belongs to the expanding mammalian facilitative glucose transporter family. Elevated GLUT1 protein expression has been observed in the majority of urothelial carcinomas, with various effects on clinicopathological parameters. Whereas malignant cells have an accelerated metabolism with increased energy requirements, the membranous expression of GLUTs is amplified. GLUT1 protein expression was evaluated in urothelial tumours of increasing grade of malignancy, supplemented by a tumour proliferation analysis. Particular attention was paid to non-invasive precursors of urothelial carcinoma. A total of 105 paraffin-embedded samples were classified (normal urothelium, low/high-grade papillary carcinoma, carcinoma in situ and invasive carcinoma). Grading and staging were conducted using the 1998 ISUP/2004 WHO criteria. The staining intensity of GLUT1 was assessed with a standard immunoreactive score (IRS). The Ki-67 index was assessed by counting positive nuclei in representative urothelial hot spots. Results showed that an increased GLUT1-IRS and mean count of Ki-67-positive cells were significantly associated with an increased grade of malignancy $(\mathrm{p}<0.0001)$, particularly in non-invasive tumours. GLUT1-IRS was significantly associated with a Ki-67-labelled proliferative fraction $(\mathrm{p}<0.0001)$. No significant association regarding tumour grade or stage was observed within the invasive carcinoma group. GLUT1 protein expression was found to be strongly correlated with increased malignant potential, particularly in non-invasive urothelial carcinomas. The increase of GLUT1 expression may reflect a preinvasive metabolic switch in terms
\end{abstract}

Correspondence to: Dr Florian Grabellus, Institute of Pathology and Neuropathology, University Hospital of Essen, Hufelandstrasse 55, Essen 45122, Germany

E-mail: florian.grabellus@uk-essen.de

Key words: GLUT1, bladder carcinoma, urothelial carcinoma, $\mathrm{Ki}-67$ of enhanced cell metabolism concomitant to known genetic alterations. A further increase in invasive carcinomas may be related to hypoxic conditions.

\section{Introduction}

Bladder carcinoma is a common cancer worldwide, with an overall incidence of 340,000 new cases each year (1). In the USA, it is the second most common malignant genitourinary disease, with an estimated 70,500 cases in 2010 (2). There were approximately 21,400 new cases in Germany in the same year (3). In Western countries, more than $90 \%$ of malignant bladder neoplasms are of urothelial origin (4). Several factors are used to predict the clinical outcome of invasive urothelial carcinoma, with multifocality, size, stage, grade and concurrent carcinoma in situ (Cis) being the most common (1). No molecular parameter has been established for routine clinical management of patients with urothelial carcinomas (5).

Glucose transporter 1 (GLUT1) belongs to the expanding mammalian facilitative glucose transporter family, which currently includes 13 members. GLUT1 is an energyindependent transport protein with a high affinity for glucose (6). It is highly expressed on endothelial cell surfaces at the blood-brain-barrier, allowing glucose entry into the brain (7), on erythrocytes (8), at the blood-ocular barrier (9) and the perineurium (10). Elevated GLUT1 mRNA levels have been reported in cancers of the pancreas, stomach, oesophagus and colon (11). In addition, GLUT1 protein expression is elevated in breast cancer (12), renal cell carcinoma (13), squamous cell carcinomas of the head and neck (14) and in carcinomas of various other sites (10). GLUT1 expression was found to be negative in normal bladder mucosa (15) and other non-neoplastic tissues (10). In contrast, a positive GLUT1 expression was reported to be present in the majority of urothelial urinary bladder carcinomas, including urothelial carcinoma of the renal pelvis (21), with varying effects on clinicopathological parameters $(15,17-21)$.

Malignant cells have an accelerated metabolism with an increased need for energy. This excess energy can be supplied by enhanced aerobic and/or anaerobic glycolytic pathways with increased glucose influx, which is associated with an 


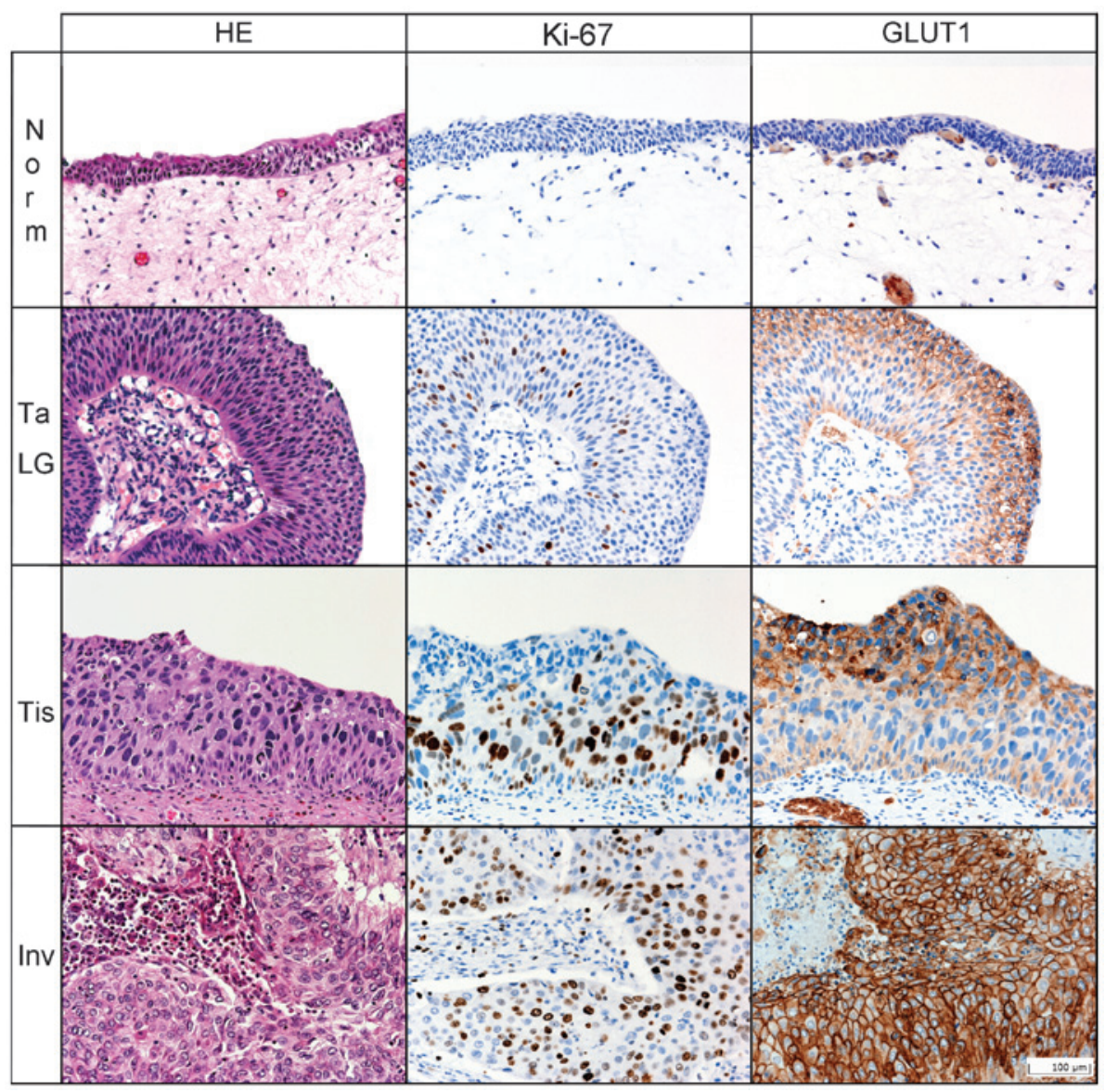

Figure 1. H\&E and immunohistochemical staining of Ki-67 and GLUT1 in normal urothelium (Norm), low-grade pTa (Ta LG; low IRS), Cis (Tis; moderate IRS) and invasive carcinoma (Inv; high IRS). Magnification, x200.

increased expression of GLUTs. Glucose is required not only for rapid ATP production, but also for accumulating biomass via the generation of precursor molecules for nucleotides, cell membranes and other components required for cell division (6). Therefore, we expect to observe marked increases in GLUT1 expression in non-invasive forms of carcinoma and further increases in invasive carcinoma relative to normal tissue.

The aim of the present study was to systematically evaluate GLUT1 protein expression in a large collection of urothelial carcinomas of increasing grade of malignancy. The analysis was supplemented by determining Ki-67-positive proliferation indices. Particular attention was paid to non-invasive precursors of urothelial carcinoma.

\section{Materials and methods}

Patients. The collection included samples from 94 men (90\%) and 11 women (10\%). The mean age of the patients was 67.8 years (range $44-87$; SD, \pm 9.8 years; men, 68 years; women, 69 years).

Sample collection. A total of 105 paraffin-embedded samples were allocated from the archive of the Institute of Pathology and Neuropathology, University Hospital of Essen, Germany. Consistent with the concept of this study, the collection included samples of increasing grade of malignancy: 20 cases of low- and 19 of high-grade papillary carcinoma (pTa), 11 of urothelial Cis, 16 of (muscle-) invasive (G2) and 20 of muscle-invasive (G3) urothelial carcinomas, as well as 19 normal urethral mucosa from transurethral resection specimens, which served as controls, from patients operated due to benign prostate hyperplasia. Surgical pathological diagnosis and grading were conducted using the 1998 ISUP/2004 WHO criteria and classified according to the UICC Classification of Malignant Tumours, seventh edition (22). Normal urothelium was defined as 5-7 layers of urothelial cells with regular architecture and absent anaplasia. Papillary carcinoma was diagnosed in cases with papillary architecture consisting of more-or-less fused and branching papillae, minor (lowgrade) or marked (high-grade) cellular and nuclear anaplasia, loss of polarity and maturation, thickening to more than seven layers and increased mitotic rate. Cases of urothelial carcinoma in situ exhibited anaplasia similar to high-grade pTa, focally not extending to the entire urothelial thickness. Normal urothelium, pTa and Cis specimens exhibited an intact basement membrane. In invasive carcinomas, tumour deposits were found beyond the basement membrane within the lamina propria or deeper.

The study was performed in accordance with the ethical standards of the Helsinki Declaration.

Immunohistochemistry. For immunohistochemistry, 4- $\mu \mathrm{m}$ sections were cut from paraffin-embedded tissue blocks. A 
Table I. Distribution of Ki-67 counts and GLUT1-IRS according to stage and grade.

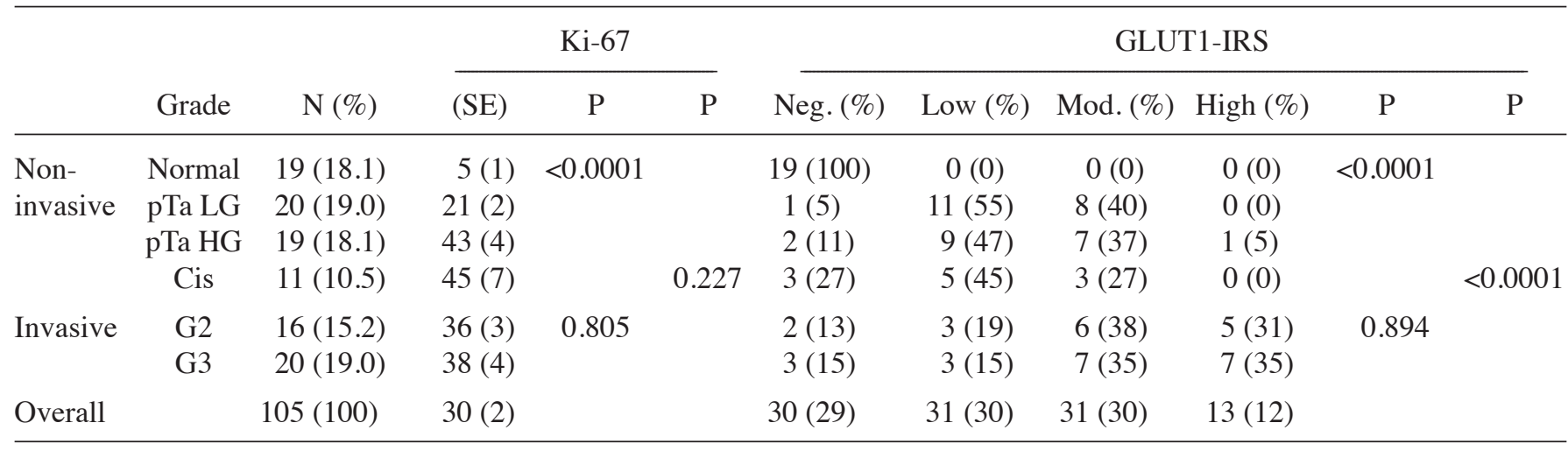

N, number of cases; SE, standard error; pTa LG, papillary urothelial carcinoma low-grade; pTa HG, papillary urothelial carcinoma high-grade; Cis, carcinoma in situ; Neg, negative; Mod, moderate.

A

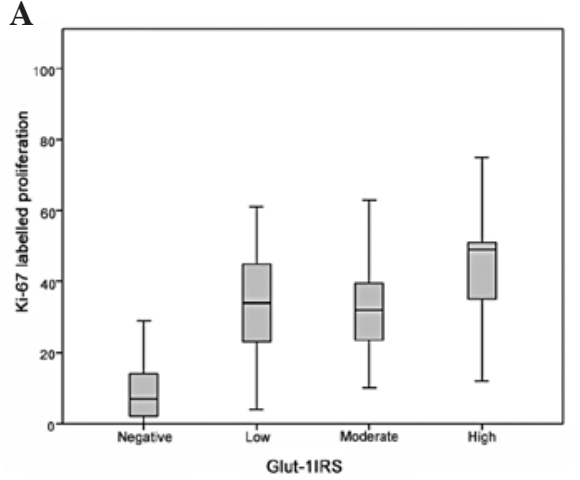

B

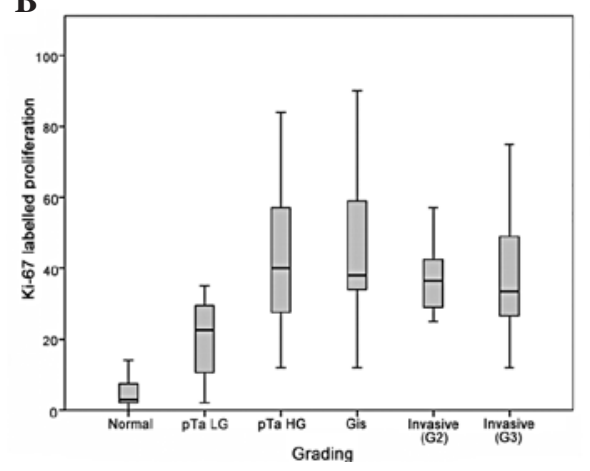

C

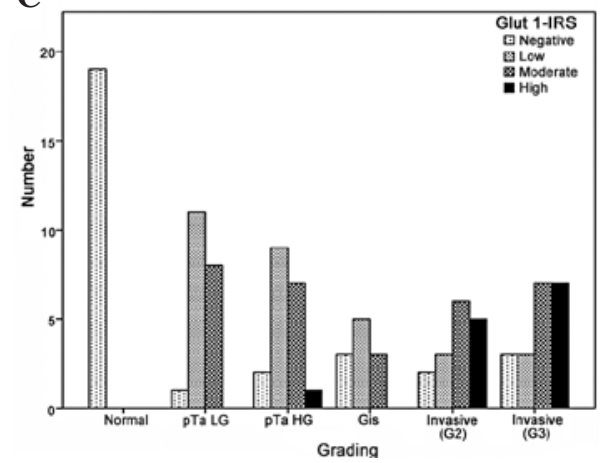

Figure 2. (A) GLUT1-IRS is correlated with the mean Ki-67-labelled proliferative fraction; (B) Mean Ki-67-labelled proliferative fraction in normal urothelium, non-invasive and invasive urothelial carcinoma; (C) GLUT1-IRS in normal urothelium, non-invasive and invasive urothelial carcinoma.

rabbit polyclonal antibody against GLUT1 (1:400; IgG, Abcam, Cambridge, UK; Fig. 1) and a mouse monoclonal antibody against Ki-67 (1:2000; IgG1, clone K-2, Zytomed Systems, Berlin, Germany; Fig. 1) were used together with a highly sensitive and specific polymer detection system utilising horseradish peroxidase (ZytoChem-Plus HRP Polymer-Kit, Zytomed Systems). Development was carried out with a permanent brown chromogenic substrate system (Permanent AEC Kit, Zytomed Systems). At the end of the procedure, sections were counterstained with hematoxylin for $5 \mathrm{~min}$.

Staining intensity of GLUT1 was assessed with an immunoreactive score (IRS) by multiplying the level of staining intensity ( 0 points, no staining; 1 point, weak staining; 2 points, strong staining) by the percentage of positive tumour cells ( $0 \%, 0$ points; $<10 \%, 1$ point; $11-50 \%, 2$ points; $51-80 \%$, 3 points; $>80 \%, 4$ points) and scored on the following scale: negative ( 0 points), weak (1-2 points), moderate (3-4 points) and strong (6-8 points). GLUT1-expression was judged as positive in cases of distinct circumferential membranous staining. Scant cytoplasmic and staining in areas of thermal injury were not considered positive. Erythrocytes served as an internal positive control.

The Ki-67 labelling index was determined by counting positive nuclei in representative urothelial hot spots. In each case, Ki-67 staining was evaluated in 100 cells. Areas showing thermal or mechanical injury were excluded from analysis.

Statistical analysis. Statistical analyses and graphical illustrations were performed using SPSS version 17.0 for Windows (SPSS Inc., Chicago, IL, USA). Univariate analysis of variance was performed for the statistical evaluation of normally distributed continuous parametric variables. Kruskal-Wallis one-way analysis of variance and the Mann-Whitney U-test were used as non-parametric methods for testing nonnormally distributed variables where appropriate. Spearman's correlation analysis was used for ordinal variables. $\mathrm{P}<0.05$ was regarded as statistically significant.

\section{Results}

GLUT1 immunohistochemistry. Increased GLUT1-IRS was significantly associated with an increased grade of tumour malignancy $(\mathrm{p}<0.0001$, Table I, Figs. 1 and $2 \mathrm{C}$ ). The GLUT1-IRS was lowest in normal urothelium and exhibited an increase in non-invasive carcinoma types $(\mathrm{p}<0.0001$; Table I). No association was found when comparing low- vs. high-grade $\mathrm{pTa}(\mathrm{p}=0.967)$, high-grade pTa vs. Cis $(\mathrm{p}=0.287)$ or low-grade pTa vs. Cis $(\mathrm{p}=0.261)$. A further marked increase 
Table II. GLUT1-IRS in correlation with Ki-67 proliferative index and distribution of Ki-67 counts and GLUT1-IRS according to invasive carcinoma stage.

\begin{tabular}{|c|c|c|c|c|c|c|c|c|c|c|c|c|}
\hline \multirow{2}{*}{$\begin{array}{l}\text { GLUT1 } \\
\text {-IRS }\end{array}$} & \multirow{2}{*}{$\mathrm{N}(\%)$} & \multirow{2}{*}{$\begin{array}{c}\mathrm{Ki}-67 \\
(\mathrm{SE})\end{array}$} & \multirow{2}{*}{$\mathrm{P}$} & \multirow{2}{*}{ Stage } & \multirow{2}{*}{$\mathrm{N}(\%)$} & \multirow{2}{*}{$\begin{array}{c}\mathrm{Ki}-67 \\
\text { (SE) }\end{array}$} & \multirow{2}{*}{$\mathrm{P}$} & \multicolumn{4}{|c|}{ GLUT1-IRS } & \multirow{2}{*}{$\mathrm{P}$} \\
\hline & & & & & & & & Neg. & Low (\%) & Mod. (\%) & High $(\%)$ & \\
\hline Negative & $30(28.6)$ & $13(3)$ & $<0.0001$ & pT1 & $1(3)$ & 40 & 0.445 & 0 & $1(100)$ & $0(0)$ & $0(0)$ & 0.295 \\
\hline Low & $31(29.5)$ & $35(3)$ & & pT2 & $14(39)$ & $37(5)$ & & $3(21 \%)$ & $4(29)$ & $4(29)$ & $3(21)$ & \\
\hline Moderate & $31(29.5)$ & $36(4)$ & & pT3 & $17(47)$ & $40(4)$ & & 0 & $0(0)$ & $8(47)$ & $9(53)$ & \\
\hline High & $13(12.4)$ & $43(5)$ & & pT4 & $4(11)$ & $27(5)$ & & $2(50 \%)$ & $1(25)$ & $1(25)$ & $0(0)$ & \\
\hline Overall & $105(100)$ & $30(2)$ & & & $36(100)$ & $37(3)$ & & & & & & \\
\hline
\end{tabular}

$\mathrm{N}$, number of cases; SE, standard error; Neg, negative; Mod, moderate.

in GLUT1-IRS was detected in the step to invasive carcinoma (Table I). No significant associations were found within the group of invasive carcinomas. No correlation was noted for tumour grade $(\mathrm{p}=0.894$; Table I) or stage $(\mathrm{p}=0.295$; Table II).

Ki-67 immunohistochemistry. The GLUT1-IRS was significantly correlated with the Ki-67-labelled proliferative fraction $(\mathrm{p}<0.0001$; Table II, Fig. 2A).

The mean number of Ki-67 positive cells was significantly correlated with the increasing grade of malignancy in noninvasive carcinoma ( $<00.0001$; Table I, Fig. 2B). However, no statistical difference was found between high-grade $\mathrm{pTa}$ and Cis $(\mathrm{p}=0.832)$. A slight decrease in the mean number of $\mathrm{Ki}-67$-positive cells was noted from Cis to invasive carcinoma, irrespective of tumour grade $(\mathrm{p}=0.805$; Table I). No correlation was found between Ki-67 staining and invasive tumour stage $(\mathrm{p}=0.445$; Table II).

\section{Discussion}

As in other types of cancer (6,10-14), GLUT1 protein expression was found to be elevated in urothelial carcinomas (15,19-21). However, findings regarding the association between GLUT1 protein expression and pathological parameters have been inconsistent (19-21).

In our study, we systematically evaluated GLUT1 protein expression in the largest collection of malignant urothelial neoplasms of increasing grade of malignancy thus far. The mean GLUT1-IRS and the mean proliferative index, estimated by Ki-67 staining, were shown to be strongly associated with an increasing grade of malignancy, from normal urothelium to non-invasive and invasive carcinoma $(\mathrm{p}<0.0001$; Table I). The GLUT1-IRS and the Ki-67 proliferative index were also significantly correlated ( $\mathrm{p}<0.0001$; Table II).

The background of enhanced GLUT1 protein expression in urothelial neoplasms of rising malignant potential may be attributable to the increased metabolism of abnormal cells. In general, these cells require greater amounts of glucose not only for energy (ATP) generation, but also for the accumulation of biomass via the generation of precursor molecules for nucleotides, cell membranes and other components required for cell division (6). This correlation is also supported by the parallel increase of the mean proliferative index in noninvasive carcinoma $(\mathrm{p}<0.0001$; Table I). Additionally, the increase in both GLUT1-IRS and Ki-67 index in non-invasive carcinoma occurs in parallel with known essential genetic alterations. Whereas FGFR3 and Ha-ras mutations play significant roles in the development of low-grade non-invasive carcinoma, a combination of $\mathrm{p} 53 / \mathrm{pRb}, \mathrm{p} 107$, and PTEN mutations and deficiencies are crucial alterations underlying high-grade urothelial carcinomas (23). These genetic changes allow cells with damaged DNA to bypass anti-proliferative and pro-apoptotic mechanisms (23).

When carcinoma cells have acquired the capacity for invasiveness, for example, at a certain level of anaplasia, genetic instability and loss of anti-tumoural inhibitors, no further acceleration of cell metabolism (glucose consumption) can be achieved. In this study, this effect may explain the failure of the GLUT1-IRS and Ki-67 indices to discriminate between tumour grades or stages following invasion. The significance of GLUT1 protein accumulation in this study appears to be as a marker of hypoxia, as the membranous GLUT1 antibody reaction was strongest in areas close to necrosis (Fig. 1). This effect was previously described and elucidated by Hoskin et al (19).

In the diagnostic setting, GLUT1 immunohistochemistry may aid the discrimination of neoplastic from non-neoplastic tissues, especially in cases of flat non-invasive urothelial lesions vs. normal urothelium and papillary carcinoma vs. papilloma. Normal urothelium consistently exhibited no GLUT1 staining (15), whereas the majority of Cis cases in the present study exhibited GLUT1 immunoreactivity. Urothelial papilloma also lacked GLUT1 immunoreactivity (16), whereas low- and high-grade pTa were, with certain exceptions, GLUT1-positive. In ambiguous cases, GLUT1 immunohistochemistry may aid in differential diagnosis in conjunction with histomorphological features and an immunohistochemical panel of CK20, Ki-67 and p53 (24).

In the clinical setting, increased glucose uptake may be visualised in malignant tumours using positron emission tomography (PET) and intravenously administered ${ }^{18}$ F-labelled 2-fluoro-2-deoxy-D-glucose (FDG) (6). Despite the excretion of FDG through the urinary tract, PET is useful for the detection of urothelial carcinomas of the urinary 
bladder when used in combination with CT imaging (25). The sensitivity of this examination may also be increased by diminishing excreted FDG activity by diuresis with furosemid or other agents (25). As in other malignant tumours (26-27), GLUT1 protein expression may be correlated with increased PET signal strength in urothelial bladder cancer, thus reflecting augmented glucose uptake and utilisation.

In conclusion, we have used a standardised scoring system to demonstrate that the GLUT1 protein expression pattern is correlated with increasing malignant potential. In addition, we have discussed possible links to underlying genetic alterations and diagnostic implications.

\section{References}

1. Lopez-Beltran A and Sauter G: Infiltrating urothelia carcinoma. In: World Health Organization Classification of Tumours - Tumours of the Urinary System and Male Genital Organs. Eble N (ed). IARC Press, Lyon, pp 93-109, 2004.

2. Jemal A, Siegel R, Xu J and Ward E: Cancer Statistics, 2010. CA Cancer J Clin 60: 277-300, 2010.

3. Husmann G, Kaatsch P, Katalinic A, Bertz J, Haberland J, Kraywinkel K and Wolf U: 3.17 Bladder. In: Cancer in Germany 2005/2006. Incidence and Trends. Robert Koch Institute (ed.) and Association of Population-based Cancer Registries in Germany (ed.) Robert-Koch-Institut, Berlin, pp 84-87, 2010.

4. Boyle P and Levin B: Bladder cancer. In: World Health Organization - World Cancer Report 2008. Boyle P and Levin B (eds.). IARC Press, Lyon, pp 444-449, 2008.

5. Kaufman DS, Shipley WU and Feldman AS: Bladder cancer. Lancet 374: 239-249, 2009.

6. Macheda ML, Rogers S and Best JD: Molecular and cellular regulation of glucose transporter (GLUT) proteins in cancer. J Cell Physiol 202: 654-662, 2005.

7. Agus DB, Gambhir SS, Pardridge WM, Spielholz C, Baselga J, Vera JC and Golde DW: Vitamin C crosses the blood-brain barrier in the oxidized form through the glucose transporters J Clin Invest 100: 2842-2848, 1997.

8. Gould GW and Holman GD: The glucose transporter family: structure, function and tissue-specific expression. Biochem J 295: 329-341, 1993.

9. Harik SI, Kalaria RN, Whitney PM, Andersson L, Lundahl P, Ledbetter SR and Perry G: Glucose transporters are abundant in cells with 'occluding' junctions at the blood-eye barriers. Proc Natl Acad Sci USA 87: 4261-4264, 1990.

10. Younes M, Lechago LV, Somoano JR, Mosharaf M and Lechago J: Wide expression of the human erythrocyte glucose transporter Glut1 in human cancers. Cancer Res 56: 1164-1167, 1996.

11. Yamamoto T, Seino Y, Fukumoto H, Koh G, Yano H, Inagaki N, Yamada Y, Inoue K, Manabe T and Imura H: Over-expression of facilitative glucose transporter genes in human cancer. Biochem Biophys Res Commun 170: 223-230, 1990.
12. Brown RS and Wahl RL: Overexpression of Glut-1 glucose transporter in human breast cancer. An immunohistochemical study. Cancer 72: 2979-2985, 1993.

13. Nagase Y, Takata K, Moriyama N, Aso Y, Murakami T and Hirano H: Immunohistochemical localization of glucose transporters in human renal cell carcinoma. J Urol 153: 798-801, 1995.

14. Mellanen P, Minn H, Grenman R and Harkonen P: Expression of glucose transporters in head-and-neck tumors. Int J Cancer 56: 622-629, 1994.

15. Chang S, Lee S, Lee C, Kim JI and Kim Y: Expression of the human erythrocyte glucose transporter in transitional cell carcinoma of the bladder. Urology 55: 448-452, 2000.

16. Lee JH, Kim YW and Chang SG: Glucose transporter-1 expression in urothelial papilloma of the bladder. Urol Int 74: 268-271, 2005

17. O'Keeffe MB, Devlin AH, Burns AJ, Gardiner TA, Logan ID, Hirst DG and McKeown SR: Investigation of pericytes, hypoxia, and vascularity in bladder tumors: association with clinical outcomes. Oncol Res 17: 93-101, 2008.

18. Zhou JT, Cai ZM, Li NC and Na YQ: [Expression of hypoxia inducible factor-1alpha and glucose transporter protein 1 in renal and bladder cancers and the clinical significance thereof]. Zhonghua Yi Xue Za Zhi 86: 1970-1974, 2006.

19. Hoskin PJ, Sibtain A, Daley FM and Wilson GD: GLUT1 and CAIX as intrinsic markers of hypoxia in bladder cancer: relationship with vascularity and proliferation as predictors of outcome of ARCON. Br J Cancer 89: 1290-1297, 2003.

20. Visca P, Sebastiani V, Pizer ES, Botti C, de Carli P, Filippi S, Monaco $\mathrm{S}$ and Alo PL: Immunohistochemical expression and prognostic significance of FAS and GLUT1 in bladder carcinoma. Anticancer Res 23: 335-339, 2003.

21. Younes M, Juarez D, Lechago LV and Lerner SP: Glut 1 expression in transitional cell carcinoma of the urinary bladder is associated with poor patient survival. Anticancer Res 21: 575-578, 2001.

22. Cheng L, Montironi R, Davidson DD and Lopez-Beltran A: Staging and reporting of urothelial carcinoma of the urinary bladder. Mod Pathol 22: S70-S95, 2009.

23. Wu XR: Biology of urothelial tumorigenesis: insights from genetically engineered mice. Cancer Metastasis Rev 28: 281-290, 2009.

24. Lindemann-Docter $\mathrm{K}$ and Knuchel R: [Update on urothelial carcinoma histopathology]. Pathologe 29: 331-338, 2008.

25. Patil VV, Wang ZJ, Sollitto RA, Chuang KW, Konety BR, Hawkins RA and Coakley FV: 18F-FDG PET/CT of transitional cell carcinoma. AJR Am J Roentgenol 193: W497-W504, 2009.

26. Hiyoshi Y, Watanabe M, Imamura Y, Nagai Y, Baba Y, Yoshida N, Toyama E, Hayashi $\mathrm{N}$ and Baba $\mathrm{H}$ : The relationship between the glucose transporter type 1 expression and F-fluorodeoxyglucose uptake in esophageal squamous cell carcinoma. Oncology 76: 286-292, 2009.

27. Usuda K, Sagawa M, Aikawa H, Ueno M, Tanaka M, Machida Y, Zhao XT, Ueda Y, Higashi K and Sakuma T: Correlation between glucose transporter-1 expression and (18)F-fluoro-2deoxyglucose uptake on positron emission tomography in lung cancer. Gen Thorac Cardiovasc Surg 58: 405-410, 2010. 